\title{
Aa. Vv., Littérature et photographie
}

\section{Francesca Forcolin}

\section{(2) OpenEdition}

\section{Journals}

\section{Edizione digitale}

URL: http://journals.openedition.org/studifrancesi/8139

DOI: 10.4000/studifrancesi.8139

ISSN: 2421-5856

\section{Editore}

Rosenberg \& Sellier

\section{Edizione cartacea}

Data di pubblicazione: 1 juillet 2009

Paginazione: 461-463

ISSN: 0039-2944

\section{Notizia bibliografica digitale}

Francesca Forcolin, «Aa. Vv., Littérature et photographie», Studi Francesi [Online], 158 (LIII | II) | 2009,

online dal 01 juillet 2009, consultato il 07 janvier 2021. URL: http://journals.openedition.org/ studifrancesi/8139; DOI: https://doi.org/10.4000/studifrancesi.8139

\section{Questo documento è stato generato automaticamente il 7 janvier 2021.}

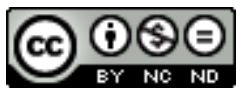

Studi Francesi è distribuita con Licenza Creative Commons Attribuzione - Non commerciale - Non opere derivate 4.0 Internazionale. 


\title{
Aa. Vv., Littérature et photographie
}

\author{
Francesca Forcolin
}

\section{NOTIZIA}

AA. VV., Littérature et photographie, sous la direction de Jean-Pierre MONTIER, Liliane LOUVEL, Danièle MÉAUX, Philippe ORTEL, Presses Universitaires de Rennes, 2008, Collection «Interférences» 2, pp. 572.

1 Il volume, che nasce dai lavori di un convegno tenutosi nel luglio 2007 al Centre National di Cerisy-la-Salle, si interroga sul ruolo che la fotografia ha giocato in relazione all'estetica letteraria. Più che essere un punto di riferimento, questa raccolta di saggi ambisce a spingere l'interesse verso un nuovo campo di ricerca.

2 Fino agli anni Ottanta, infatti, cioè fino agli studi principalmente di Roland Barthes e Philippe Dubois, la critica letteraria ha ignorato il decisivo impatto della fotografia sull'estetica, sulla società, sulla politica e letteratura di due secoli. A partire dalla creazione del termine, avvenuta in Francia nel 1839 grazie alle sperimentazioni di Joseph N. Niépce e di Louis Daguerre, seguite dalla calotipia con William Fox Talbot, la fotografia è stata al centro di discussioni, polemiche, conflitti, in quanto "rivoluzione invisibile" (secondo la definizione di Philippe Ortel) che promuoveva il dominio della materia a scapito del tentativo di mimesi del reale operato in letteratura e in pittura. Proprio Baudelaire, il maggior sostenitore della "modernità", aveva contraddittoriamente sottolineato l'incolmabile divario tra la poesia ("l'arte eterna") e la fotografia, intesa come ambizioso simbolo del progresso.

Considerata per molto tempo più vicina alla scienza che all'arte, la fotografia, "immagine industriale", segnò una cesura nella storia dell'umanità: diede vita ad una diversa concezione dell'opera d'arte, a un cambiamento nella visione del reale (da cui nacque il realismo e la sua antitesi, il genere fantastico); intervenne nell'evoluzione della letteratura di viaggio, nella rappresentazione dell'“altro"; modificò la concezione del tempo in seguito alla decomposizione del movimento nelle opere di Eadweard Muybridge; ma soprattutto fu indispensabile per la rappresentazione dell'identità 
(sessuale, razziale) e per l'evoluzione dell'io, dell'intimità, della memoria. Ed è sulla ridefinizione del soggetto, del moi, che si muovono gran parte delle opere di autofiction "photo-littéraires" analizzate nei contributi raggruppati in questo volume, di ricercatori internazionali; riflessioni teoriche che intendono sottolineare come l'opera letteraria e la fotografia non abbiano seguito linee parallele senza mai entrare in contatto, come per molto tempo si è creduto, ma che si siano congiunte e legate dando vita a interazioni non solo feconde, bensì rivoluzionarie.

Dopo l'Avant-propos di Jean-Pierre MONTIER (pp.7-14), i curatori hanno suddiviso il volume in cinque capitoli che esaminano l'evoluzione della fotografia, il dialogo tra lo spazio della pagina scritta e lo spazio dell'io, il rapporto tra narrativa e immagine, il territorio del visibile e del leggibile. Nella parte centrale del volume si trova il cahier couleur, una ventina di pagine comprendenti 48 fotografie descritte nei saggi, dalla più antica - Point de vue pris d'une fenêtre du Gras di Nicéphore Niépce, 1827 - alle ultime sperimentazioni sulla luce con l'utilizzo di filtri (Michel Séméniako).

5 La prima sezione, «Convergences» (pp.17-168), raccoglie i saggi di Philippe ORTEL su Trois dispositifs photo-littéraires. L'exemple symboliste; di Paul EDWARDS su Tendances nationales et tendances économiques dans la constitution de l'objet photolittéraire; di Monique SICARD, Photographie: quel récit des origines?; Marta CARAION, Texte-photographie: la verité selon la fiction; Paul-Louis ROUBERT, Alfred Tonnellé et la photographie, ou les faillites de l'harmonie; Claire BUSTARRET, Photographie et autographie: statut paradoxal du fac-similé; Paul LÉON, L'écrivain et ses images, le paratexte photographique; Leszek BROGOWSKI, Le documentaire: dispositif photographique, dispositif littéraire; Pierre-Henry FRANGNE, Photographie et littérature de montagne ou le sublime contemporain.

Nell'ambito di «Le photographe à l'œuvre» (pp.169-326), troviamo gli studi di Christelle REGGIANI, Subjectivité littéraire et photographie: la naissance du point de vue; Lawrence GASQUET, "On dit que nous autres photographes, nous sommes au mieux une race d'aveugles»: l'image du photographe dans l'œuvre littéraire de Lewis Carrol; Émile PITON-FOUCAULT, Merveille et monstruosité de l'image photographique dans les "Rougon-Macquart" d'Émile Zola; Marie-Hélène BОвLET, "L'agrandissement" ou "Le balcon (détail)": le roman-photo à la Mauriac; Laurence PETIT, «Spectres de Kath»: "La Photographie" "au négatif» de Penelope Lively; Jean ARROUYE, La vision oblique; Anne-Cécile GUILBARD, Le roman du regardeur en 1980 (Roland Barthes et Hervé Guibert); Isabelle RousSEL-GILLET, Les paradoxes de la photographie chez Ernaux et Le Clézio; Bernard LAFARGUE, Le temps des avatars virtuels: entre la vie éternelle de Houellebecq et la vie divine de Sollers; Danièle mÉAuX, L'écriture à l'épreuve de l'image enregistrée. Particolarmente interessante è il testo di Roussel-Gillet, che analizza l'apparente diversa funzione della fotografia nelle opere di Ernaux e Le Clézio: immagini sovresposte di vestiti abbandonati al suolo come espressione di una libido in atto (nella prima) e paesaggi africani che rappresentano un passato ancestrale (nel secondo), sono accomunati dalla memoria, dal passato e dall'affezione verso gli oggetti.

7 Nel terzo capitolo, «Espaces du livre, espaces de soi» (pp.327-412), sono raccolti gli articoli di Évelyne ROGNIAT, Chambres d'écho: propos sur la collection "Carnets de voyages"; Jan BAETENS, La lecture narrative de l'image photographique; Magali NACHTERGAEL, Roland Barthes et les artistes des mythologies individuelles: la création de soi par la photographie entre 1970 et 1975; Chloé CONANT, Histoire d'images et de textes: les cuvres photo-fictionnelles de Sophie Calle et d'Édouard Levé; Cécile CAMART, Les stratégies éditoriales de Sophie Calle: livres de photographies, photo-roman, livres d'artiste; Nancy PEDRI, Le silence photographique, un 
geste provocateur, dove si prende in considerazione la narrazione silenziosa della fotografia, che attraverso gesti visivi permette l'espressione del moi, con un riferimento particolare al racconto autobiografico Crybaby di Janice Williamson; Laurence PERRIGAULT, La photographie comme déclencheur de la mémoire dans "Le royaume des voix" d'Antonio Muñoz Molina et "La ferme du Garet" de Raymond Depardon.

8 La penultima sezione, dal titolo «Territoires du visible et du lisible» (pp.413-516), comprende i contributi di Jean-Pierre MONTIER, Et vint "La vue"; Daniel GROJNOwSKI, Eugène Atget, artisan photographe et auteur d'avant-garde; Jay BOCHNER, Textes vus chez Walker Evans; Véronique MONTÉMONT, Dites voir (sur l'ekphrasis'); Dervila cooKE, Maspero et Frantz: voyages vers l'autre en photographie et en texte; Laurence GUYON, "C'que c'est beau la photographie!»; Christine BUIGNET, Tropismes photographiques. Indicible et Inimageable. L’ultimo capitolo, «Zoom et Aperçus» (pp.517-546), raccoglie gli studi di Jérôme тHÉLoT, Notes sur un portrait de Baudelaire; Paul EDWARDS, Brève présentation de l'Ouphopo, in cui si espongono brevemente gli obiettivi dell'“Ouvroir de photographie potentielle" il quale, nato nel 1995, segue il percorso iniziato negli anni Sessanta da François Le Lionnais con l'Oulipo; Ronald shusterman, Polarités: la photographie entre littérature et sensation; un Entretien de Danièle mÉAUX avec Michel sÉMÉNIAKO, Genèse d'un projet: du texte à l'image - À propos d'“Exil", photographies de Michel Séméniako, textes de Louise L. Lambrichs.

Chiude il volume una breve presentazione degli autori. 